\title{
A Fault-tolerant Algorithm for Decentralized On-line Quorum Adaptation*
}

\author{
Mark Bearden \\ Bell Laboratories \\ Lucent Technologies, Murray Hill, New Jersey \\ mbearden@research.bell-labs.com
}

\begin{abstract}
A quorum-based distributed mutual exclusion protocol requires each processor in a distributed system to obtain permission from a quorum of processors before accessing a resource that cannot be concurrently shared. To prevent failed quorum members from blocking access to the resource, it is desirable to remove failed processors from quorums when failures are detected. This work addresses the problem of adapting quorums on-line, while a quorum-based mutual exclusion protocol continues to operate. To preserve the quorum intersection property that is required for mutual exclusion safety, it is necessary to coordinate changes made to the quorum data structures of different processors. A solution is given in the form of QADAPT, a decentralized algorithm that guarantees safe adaptation of quorums when processors fail. QADAPT enables any set of quorum adaptations that do not violate the quorum intersection property, and enables any set of faulty processors to be removed from quorums. QADAPT has optimal message passing cost and tolerates any number of processor (halting) failures. A distributed system model is assumed that provides only point-to-point messages with no message ordering. Results from an implementation show that the algorithm's execution time scales well in a system containing up to fifty networked workstations. Extensions of this work include on-line adaptation of quorums that are used to maintain replica consistency in distributed databases.
\end{abstract}

\section{Introduction}

Mutual exclusion is the classic problem [13] of coordinating access among several users to a shared critical resource. The coordination ensures safety, such that no two users access the critical resource simultaneously, and progress, such that any processor requesting access to the resource, and waiting a sufficiently long time, eventually obtains access. In the distributed form of the mutual exclusion problem, the users are a group of networked processors that coordinate access to the critical resource by exchanging messages among themselves. This work

\footnotetext{
*Published in the Proceedings of FTCS-28, June 23-25, 1998 in Munich, Germany. This work was supported in part by NSF Grant CCR-9257973 while the first author was a student in the Electrical and Computer Engineering Department at Carnegie Mellon University. The authors wish to thank Mark Stahl and Nina Banerjee for their helpful comments on this work. (C) 1998 IEEE. Personal use of this material is permitted. However, permission to reprint/republish this material for advertising or promotional purposes or for creating new collective works for resale or redistribution to servers or lists, or to reuse any copyrighted component of this work in other works, must be obtained from the IEEE. Contact: Manager, Copyrights and Permissions, IEEE Service Center, P. O. Box 1331, Piscataway, NJ 08855, USA. Telephone: +Intl. 908-562-3966.
}

\author{
Ronald P. Bianchini, Jr. \\ Electrical and Computer Engineering Department \\ Carnegie Mellon University, Pittsburgh, Pennsylvania \\ rpb@ece.cmu.edu
}

considers the class of solutions to distributed mutual exclusion that use quorums $[14,15,25,31,33]$. These protocols require each processor to obtain permission from a set of processors called a quorum prior to accessing the critical resource. Each processor is assigned a distinct quorum such that the intersection of any two processors' quorums is not empty. This quorum intersection property ensures that if any two processors attempt to access the resource concurrently, then both must obtain permission from some common processor, contained in both processors' quorums. Thus it is possible via the common processor to prevent two processors from accessing the critical resource simultaneously.

A quorum assignment is a set of quorums that consists of one quorum for each processor that can request access to the critical resource. A quorum assignment is valid if and only if the quorums contained in the assignment satisfy the quorum intersection property. Numerous algorithms for generating valid quorum assignments have been suggested $[2,9,10,25,26,29]$. Sanders' generalized form of the quorum-based mutual exclusion problem, given in [31], provides a distributed mutual exclusion algorithm that solves mutual exclusion when used with any valid quorum assignment. We assume the use of Sanders' distributed mutual exclusion algorithm, which is outlined briefly below.

Each processor that wishes to access the critical resource sends a request message to each processor in its quorum and then waits to receive grant messages in response. When a processor has received a grant message from every member of its quorum, it can safely access the critical resource. When a processor gives up access of the critical resource, it sends a release message to all of the processors that previously sent it grant messages. Mutual exclusion is ensured by allowing each processor to grant permission to only one processor at a time. Once a grant message has been sent by processor $x$ to processor $y$, processor $x$ is locked, such that $x$ sends no other grant messages before it receives a release message from $y$. Full details of the mutual exclusion algorithm are given in [31], including extensions that prevent deadlock when processors make concurrent requests, or fail before sending a release message to a locked processor.

\subsection{Impact of Faulty Processors on Quorums}

This work considers how to ensure mutual exclusion progress when processors contained in quorums fail by halting. It is assumed that a faulty processor will not send a grant message in response to a request message. Since a processor must receive a grant message from each member of its quorum before accessing the critical resource, faulty 
processors in quorums threaten progress. Ensuring progress requires either that faulty processors are masked by the quorum assignment, or else that the quorum assignment is adapted to remove faulty processors from quorums.

Solutions such as those in $[8,29]$ mask faulty processors by requiring multiple processors in the intersection of any two quorums. For example, if up to $f$ processors can fail simultaneously, then guaranteeing progress requires at least $2 f+1$ processors in the intersection of any pair of quorums. A processor can access the critical resource if grant messages are received from all but $f$ members of the processor's quorum [8]. An alternative form of fault masking assigns multiple quorums, instead of a single quorum, to each processor. Each processor sends request messages to the members of multiple quorums $[15,24,33]$, and the processor is allowed to access the resource if it obtains grant messages from all of the processors in at least one of its quorums. This ensures progress whenever at least one of each processor's quorums contains no faulty processors. By increasing the size and/or number of quorums assigned to each processor, a masking approach increases the communication cost of requesting access to the critical resource. Communication overhead is minimal if the quorum assignment has only a single quorum per processor, and if the intersection of any two given quorums contains only one processor [25].

Rather than masking faulty processors, this work considers the adaptation of quorums when failures are detected. In this approach, if processor $x$ fails when $x$ is the only processor contained in the intersection of quorums $Q^{j}$ and $Q^{k}$, then $x$ is removed from $Q^{j}$ and $Q^{k}$, and some non-faulty processor other than $x$ is added to both $Q^{j}$ and $Q^{k}$. Quorum adaptation is preferable to fault masking if faulty processors can be detected, and if the occasional communication and delay needed for quorum adaptation are acceptable costs.

\subsection{Related Research}

A body of previous research addresses the fault-tolerance of quorum assignments. Probabilistic approaches such as those in $[1,2,19,22,27,29]$ seek to determine the likelihood that progress is satisfied, given that a non-adaptive, or static, quorum assignment is used. These approaches assume that processors fail with a known probability, and compute the resulting probability that failures will block progress. This assists in a priori selection of static quorum assignments that maximize the probability of ensuring progress. Other research has focused on deterministic guarantees of fault-tolerance. The work in [8] generates static quorum assignments that are guaranteed to mask up to a predetermined number of failed processors. Adaptive deterministic fault-tolerance is provided by the algorithms in $[2,9,10,26,31]$, that adapt quorum assignments after the detection of processor failures. Each processor autonomously computes the list of processors in its own quorum, based upon changing local information about which processors are faulty. Another approach to adapting quorum assignments uses dynamic voting [17,18,23]. All known previous approaches to quorum adaptation limit the set of possible quorum adaptations by restricting the size and/or topology of quorums that result after adaptation, or by requiring that some particular subset of the processors remain non-faulty. Eliminating these restrictions is a motivation of this work. A quorum adaptation approach is given that operates with any method for determining quorum assignments, tolerates any number of simultaneously faulty processors, and does not require the mutual exclusion protocol to be halted when quorums are adapted.

\subsection{The On-line Quorum Adaptation Problem}

It is assumed, as in [31], that each processor in a distributed system stores in local memory a list of the processors in its quorum. When quorums are adapted to ensure progress, processors update their local memories to reflect the changes to their quorums. Although faulty processors contained in the quorums of a valid quorum assignment cannot threaten safety, adapting the quorum assignment can threaten safety. Even if the quorum assignment is valid before and after quorums are adapted, it is possible for processors to update their local memories in an order such that the quorum intersection property is temporarily violated.

Figure 1 illustrates a sequence of quorum adaptations that temporarily threaten safety. Two quorums, $Q^{j}$ and $Q^{k}$, are given below in (a), for two processors $j$ and $k$ (not shown). Initially, quorums $Q^{j}$ and $Q^{k}$ are part of a valid quorum assignment, and the intersection of $Q^{j}$ and $Q^{k}$ contains processor $x$. Subsequently, $x$ is removed from $Q^{j}$ and $Q^{k}$, and is replaced with processor $y$ in both quorums. The resulting state of $Q^{j}$ and $Q^{k}$, which also satisfies the quorum intersection property, is given in (c). Suppose that the modifications to $Q^{j}$ and $Q^{k}$ are not made simultaneously, such that $x$ is replaced with $y$ in quorum $Q^{k}$ before $Q^{j}$ is modified; then the quorums temporarily have the arrangement given in (b). The quorum assignment is temporarily invalid, and safety can be violated if the mutual exclusion algorithm executes. Other possible sequences of quorum modifications never result in a state such that $Q^{j}$ and $Q^{k}$ have an empty intersection. For example, it is possible to transition from the state in (a) to the state in (c) through the states shown in (d) and (e), by adding $y$ to both $Q^{j}$ and $Q^{k}$ before $x$ is removed from either $Q^{j}$ or $Q^{k}$.

This work addresses the problem of adapting the quorums in a quorum assignment such that any combination of faulty processors do not threaten progress. It is assumed that quorums are adapted on-line, while a quorum-based mutual exclusion protocol executes without interruption. The mutual exclusion protocol uses the quorum assignment provided by a quorum adaptation protocol. Over time, the quorum assignment is modified to remove faulty proces-

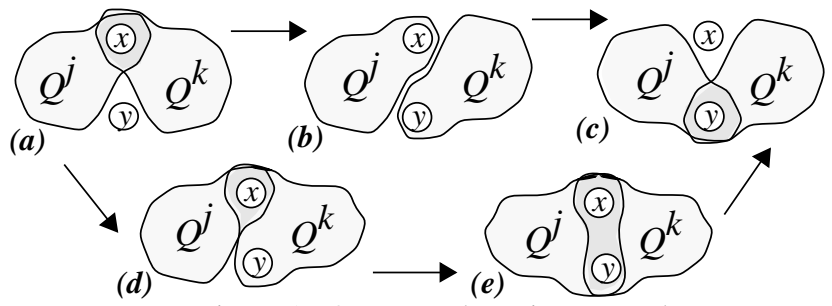

Figure 1: Quorum adaptation example. 
sors from quorums. Because the mutual exclusion protocol is not halted, safety requires that the modifications made to quorums are ordered such that the quorum intersection property is continuously preserved. This problem is called on-line quorum adaptation, abbreviated $O Q A$. The work in [4] introduced the OQA problem and considered the order in which quorum modifications must occur to preserve the quorum intersection property. This work gives a solution to OQA in the form of a distributed $O Q A$ algorithm. An OQA algorithm allows each processor to locally determine a desired set of modifications to its local quorum. Each desired quorum modification is delayed until the OQA algorithm determines that the modification cannot lead to a safety violation. Although the focus of the discussion is fault-tolerance, it is not necessary to assume that quorums are modified only to remove faulty processors. For example, an OQA algorithm enables on-line rearrangement of quorums to balance processor load, or to include processors that recover from failures or are added to the system.

The OQA algorithm presented here does not restrict the set of valid quorum assignments that may result after quorum adaptation. Any valid quorum assignment can be replaced with any other desired valid quorum assignment. A general distributed processing model is used which assumes that any number of processors may simultaneously fail or recover, and that processors communicate only by exchanging unordered messages. The paper is organized as follows. Section 2 gives a formal statement of the problem and system model. Section 3 gives a distributed OQA algorithm called QADAPT. Section 4 discusses execution costs of QADAPT, and Section 5 gives a summary of the work.

\section{System Execution Model}

The following definitions are used to present the OQA algorithm. The set of processors in a distributed system is denoted $\mathrm{S}$. The set of non-faulty processors in $\mathrm{S}$ is $U P(\mathrm{~S})$. Individual processors are allowed to leave and rejoin $S$ any number of times, and processors may fail and recover while in $\mathrm{S}$; thus the values of $\mathrm{S}$ and $U P(\mathrm{~S})$ vary over time. The system is stable during an interval if no changes occur to $\mathrm{S}$ or $U P(\mathrm{~S})$ during the interval. Processor failures are assumed to be fail-stop [32]. Each processor has private storage containing information about its own status and the status of other processors.

Without loss of generality, there is one critical resource associated with $S$. Some of the processors in $U P(\mathrm{~S})$ execute a distributed quorum-based mutual exclusion protocol to share the resource. The mutual exclusion protocol uses the quorum assignment, called $Q A$, that is provided by the OQA algorithm. For each processor $p$ that executes the mutual exclusion protocol, $Q^{p}$ denotes the quorum of $p$ that is identified in $p$ 's local storage. Each $p$ also stores a boolean flag called mutex ${ }^{p}$ that indicates whether or not $p$ is currently allowed to send request messages to processors in $Q^{p}$. The quorum assignment $Q A$ is defined by $\left\{Q^{x} \mid x \in U P_{\mathrm{S}} \wedge\right.$ mutex $\left.^{x}=\mathrm{TRUE}\right\}$. The set $\mathrm{S}_{\text {mutex }}$ is defined by $\left\{x \mid Q^{x} \in Q A\right\} . \mathrm{S}_{\text {mutex }}$ is the set of processors that "know" they can safely request access to the critical resource using quorum assignment $Q A$.

The configuration of $S$ is the subset of the system state that determines whether or not the mutual exclusion protocol is guaranteed to provide safety. When $U P(\mathrm{~S})$ equals $\{a, \ldots, z\}$, the configuration is defined by the set of duples $\left\{\left(Q^{a}\right.\right.$, mutex $\left.^{a}\right), \ldots,\left(Q^{z}\right.$, mutex $\left.\left.^{z}\right)\right\}$. The value of the configuration changes when quorums are adapted and when processors fail and recover, or leave and join $S$.

During execution, the system acquires a discrete sequence of configuration values $C_{0}, C_{1}, C_{2}, \ldots$, where $C_{0}$ is the configuration value for the initial system state. An event refers to the transition from one configuration value to a successive configuration value. Each event changes the status of one processor $p$, such that

- $p$ changes the value of mutex $^{p}$, or

- $p$ adds (removes) exactly one element to (from) $Q^{p}$, or

- $p$ joins $U P(\mathrm{~S})$ or leaves $U P(\mathrm{~S})$.

It is assumed that system actions which are not defined as events can occur at any time relative to events; these include the sending of messages, or changes to system state that do not modify the configuration of S. Four special types of events - called Start, Stop, Add, and Del eventsare defined below in Table 1. A subscript $i$ is associated with an event (i.e., ' $A d d_{i}\left(Q^{p}, x\right)$ ') to indicate that the event corresponds to the transition between configurations $C_{i-1}$ and $C_{i}$. An event that occurs at an unspecified transition is denoted without a subscript.

The order in which Start, Add, and Del events occur is assumed to be determined by an OQA algorithm. An OQA algorithm ensures safety by controlling the order in which events of these three types occur. The quorum intersection property for the quorums in quorum assignment $Q A$ can only be violated if these events are performed in the wrong order [4], as illustrated in Figure 1.

\subsection{Communication and Failure Detection}

Processors communicate by exchanging messages over a network that is logically fully connected. Each processor has a unique identifier and can correctly identify the sender of any received message. Communication is loosely synchronous: Processors may execute at different speeds, and message delivery delays may be non-deterministic, but there exist known upper bounds on the relative processing speed of any two processors, and on delay of delivering a sent message. Messages exchanged between non-faulty processors are reliably delivered. This implies that the network does not partition [30]. Section 5 discusses relaxing this assumption. Messages that are lost due to processor failures can be detected by time-outs. When a non-faulty processor $p$ sends a message $m$ to $q$, and $m$ is not delivered because $q$ is faulty or not in $\mathrm{S}$, then $\operatorname{lost}(m)$ denotes the

\begin{tabular}{|l|l|}
\hline Notation & \multicolumn{1}{|c|}{ Event Description } \\
\hline \hline Start $(p, K)$ & $\begin{array}{l}\text { Processor } p \text { with } Q^{p} \text { equal to } K \text { joins } \mathrm{S}_{\text {mutex }} \text { by set- } \\
\text { ting } \text { mutex }^{p} \text { equal to TRUE }\end{array}$ \\
\hline Stop $(p)$ & Processor $p$ leaves $\mathrm{S}_{\text {mutex }}$ by failing or by exiting S \\
\hline$A d d\left(Q^{p}, x\right)$ & Processor $p \in \mathrm{S}_{\text {mutex }}$, adds processor $x$ to $Q^{p}$ \\
\hline $\operatorname{Del}\left(Q^{p}, x\right)$ & Processor $p \in \mathrm{S}_{\text {mutex }}$, removes processor $x$ from $Q^{p}$ \\
\hline
\end{tabular}


moment when $p$ determines $m$ will not be delivered.

A failure suspector mechanism exists at each processor. The failure suspector at a non-faulty processor provides a local suspicion of which other processors are currently non-faulty. The failure suspector at $p$ maintains a list of processors, $U P^{p}$, that are currently believed by $p$ to be in $U P(\mathrm{~S})$. If processor $x$ is not in $U P^{p}$, this means $p$ suspects that either $x \notin \mathrm{S}$, or else $x \in \mathrm{S}$ but $x \notin U P(\mathrm{~S})$. The failure suspectors in $\mathrm{S}$ satisfy two correctness requirements:

- FS-Stabilization: Given a constant $\mathrm{T}_{F S \text {-Stabilize }}$, if the system is stable during some interval $\left(t, t+T_{\text {stable }}\right)$ such that $T_{\text {stable }}>T_{F S \text {-Stabilize }}$, then during the interval $\left(t+T_{F S \text { Stabilize }}, t+T_{\text {stable }}\right)$ it must be true that $U P^{p}=U P(\mathrm{~S})$ for each $p \in U P(\mathrm{~S})$

- FS-Comm-Accuracy: At time lost(M), for message $M$ sent by $p$ to $q, q \notin U P^{p}$ and $q \notin U P(\mathrm{~S})$ must both be true.

FS-Stabilization allows that the failure suspectors of non-faulty processors may temporarily disagree about which processors are non-faulty, but requires that they must eventually agree on the actual set of non-faulty processors. No system-wide order is imposed on the changes that occur to failure suspector output, nor must the different processors suspect the same set of changes to $U P(\mathrm{~S})$. FS-Comm-Accuracy means that if $p$ sends a message $M$ to faulty processor $q \notin U P(\mathrm{~S})$, then when message $M$ is determined to be lost, $p$ must also suspect $q \notin U P^{p}$. Thus $p$ 's suspicion of $q$ 's status is accurate, and corresponds to $q$ 's actual status, only when $p$ communicates directly with $q$. Processor $p$ can force an accurate suspicion of $q$ by attempting to communicate with $q$. The system provides no ordering of message deliveries with respect to changes in the output of failure suspectors. Failure suspectors can be efficiently implemented using communication that is intrinsic to the mutual exclusion algorithm [28], or they can utilize separate membership [7] or system diagnosis [6] services.

\subsection{OQA Algorithm Operation}

An OQA algorithm ensures that the quorum assignment $Q A$ eventually equals a desired quorum assignment, i.e. some quorum assignment that contains no faulty processors. Each processor $p$ has a local variable called $Q A$-desired ${ }^{p}$ that indicates the value $p$ currently expects to be the correct value for $Q A$. $Q A$-desired ${ }^{p}: Q^{x}$ denotes the quorum that $p$ expects to be currently correct for processor $x$. $Q A$-desired ${ }^{p}$ is expected to take on a succession of values over time, as $p$ detects the failure of other processors using the output of $p$ 's failure suspector. Since failure suspectors of different processors may temporarily disagree, $Q A$-desired ${ }^{p}$ may at any given time disagree with $Q A$-desired ${ }^{q}$, for any $p$ and $q$. However, FS-Stabilization ensures that the processors must eventually agree on a single value for $Q A$-desired ${ }^{p}$. The desired quorum assignment, denoted QA-desired and defined by $\left\{Q A\right.$-desired $\left.{ }^{x}: Q^{x} \mid x \in U P(\mathrm{~S})\right\}$, may be at times invalid, but it must eventually equal a valid assignment. It is assumed that the value of $Q A$-desired ${ }^{p}$ is computed by some function $F\left(U P^{p}\right)$, where $F$ is a deterministic function defined over all possible values of $U P^{p}$, and $Q A$-desired ${ }^{p}: Q^{x}=\varnothing$ is always true for any $x \notin U P^{p}$.

Each non-faulty processor is assumed to continuously execute an OQA algorithm. Whenever $Q A$-desired ${ }^{p}: Q^{p}$ has a value different from the current value of $Q^{p}$, the OQA algorithm attempts to modify $Q^{p}$ such that $Q^{p}$ eventually equals $Q A$-desired ${ }^{p}: Q^{p}$. Processor $p$ is allowed to execute the mutual exclusion protocol only when mutex ${ }^{p}$ equals TRUE. When $p$ joins $\mathrm{S}$ or recovers from a failure, mutex $^{p}$ initially equals FALSE. The OQA algorithm determines when the mutex $^{p}$ flag at $p$ can be safely set to TRUE. $Q^{p}$ is not required to have a non-empty intersection with other quorums when $p \notin \mathrm{S}_{\text {mutex }}$, but there must exist no $q \in \mathrm{S}_{\text {mutex }}$ such that $Q^{q} \cap Q^{p}=\varnothing$ when the OQA algorithm sets mutex $^{p}$ to TRUE.

An on-line quorum adaptation algorithm guarantees the following properties:

- OQA-Safety: QA always represents a valid quorum assignment;

- OQA-Progress: It is always true that the system will eventually reach a future state such that at each non-faulty $p, Q^{p}$ contains only non-faulty processors, and $Q^{p}=Q A$-desired ${ }^{p}: Q^{p}$.

OQA-Safety requires that the quorum assignment $Q A$ is always valid, although the quorums in $Q A$ are allowed at times to contain faulty processors that temporarily block progress. Although $Q A$-desired can temporarily represent an invalid quorum assignment, OQA-Progress requires that the non-faulty processors will eventually adapt their quorums such that the quorum assignment $Q A$ equals $Q A$-desired and permits mutual exclusion progress.

\subsection{System Stabilization Assumptions}

Satisfying OQA-Progress requires the following "always-eventually" system stability assumption. It is always true that the system will eventually remain stable during some interval of duration $D$, where $D$ is an a priori given constant large enough that

$$
T_{F S \text {-Stabilize }}+T_{Q A \text {-desired }}+T_{O Q A}<D
$$

is satisfied. $\mathrm{T}_{F S \text {-Stabilize }}$ is given in Section 2.1 as the delay for failure suspectors to agree on the correct set of non-faulty processors. $T_{Q A \text {-desired }}$ is the maximum delay required for every $p$ to agree on the same value for $Q A$-desired ${ }^{p}$, such that each $Q A$-desired ${ }^{p}: Q^{p}$ contains no faulty processors, after the failure suspectors have agreed on the non-faulty processors. $\mathrm{T}_{O Q A}$ is the maximum delay for the OQA algorithm to achieve $Q A=Q A$-desired, once the processors have agreed on the desired quorum assignment. Informally stated, the processors must eventually agree on a valid desired quorum assignment $Q A$-desired that will permit progress, and must agree long enough for an OQA protocol to ensure the value of $Q A$ "catches up" with the value of $Q A$-desired. If the mean time between failures or system reconfigurations is large compared to the execution time of an OQA and failure suspector protocols, then this assumption is easily met.

\section{The QADAPT Algorithm}

This section presents an OQA algorithm called 
QADAPT. QADAPT is fully decentralized and fault-tolerant, such that all processors execute the same set of instructions and share equal responsibility for solving OQA. While any processor remains non-faulty, OQA-Safety and OQA-Progress are guaranteed. Using the value of $Q A$-desired, QADAPT determines a set of events that will satisfy OQA-Progress while ensuring that the events are executed in an order that satisfies OQA-Safety.

When the value of $Q A$-desired changes, each processor $p$ independently determines a set of $\operatorname{Add}\left(Q^{p},-\right)$ and $\operatorname{Del}\left(Q^{p},-\right)$ events that will modify $Q^{p}$ so that it equals the most recent value of $Q A$-desired ${ }^{p}: Q^{p}$. It is proven in [4] that $A d d$ and Stop events may occur at any time relative to other events without jeopardizing OQA-Safety, but that any Del or Start events must be partially ordered with respect to other Del, Start, and $A d d$ events. A desired $\operatorname{Add}\left(Q^{p}, x\right)$ event is executed by processor $p$ as soon as $x$ is added to $Q A$-desired ${ }^{p}: Q^{p}$. A desired $\operatorname{Del}\left(Q^{p}, x\right)$ event must be delayed by $p$ until $p$ knows that $x$ cannot be the only processor in $Q^{p} \cap Q^{q}$, for any $q \in \mathrm{S}_{\text {mutex }}$. A desired $\operatorname{Start}(p, K)$ event must be delayed until processor $p$ knows that $Q^{p} \cap Q^{q} \neq \varnothing$ for every $q \in \mathrm{S}_{\text {mutex }}$. For processor $p$ to determine that a $\operatorname{Del}\left(Q^{p},-\right)$ or $\operatorname{Start}(p,-)$ event will not violate OQA-Safety, $p$ must first learn that some set of Add or Start events have previously occurred at processors other than $p$ [4]. This knowledge is established by the exchange of interprocessor messages that contain quorum tokens, as described below.

After any $\operatorname{Add}\left(Q^{p}, x\right)$ event is executed at $p, p$ sends messages to other processors to inform them that the $\operatorname{Add}\left(Q^{p}, x\right)$ event has occurred. The messages sent by $p$ convey quorum tokens that identify the occurrence of Add events. An $x$-token sent by $p$ is a quorum token that indicates $p$ has added $x$ to $Q^{p}$. A quorum token sent by $p$ via a message that is received by $q$, is said to be held by $q$, until $q$ returns the token to $p$ via a message sent to $p$.

After an $\operatorname{Add}\left(Q^{p}, x\right)$ event, $p$ sends an $x$-token to each $q$ such that $x$ is in $Q A$-desired ${ }^{p}: Q^{q} \cap Q^{p}$. Because $Q A$-desired ${ }^{p}$ and $Q A$-desired ${ }^{q}$ must eventually agree, $p$ can anticipate that each processor in $\left\{q \mid x \in Q A\right.$-desired $\left.{ }^{p}: Q^{q}\right\}$ will eventually desire to add $x$ to $Q^{q}$. The intersection of $Q A$-desired ${ }^{p}: Q^{q} \cap Q A$-desired ${ }^{p}: Q^{p}$ and $Q^{q}$ must eventually contain some processor $x$, for each $q \neq p$, such that $q$ receives an $x$-token sent by $p$. If $p$ subsequently desires to remove $x$ from $Q^{p}$, because $x$ is removed from $Q A$-desired ${ }^{p}: Q^{p}$, then $p$ requests that processors return any $x$-tokens they hold from $p$.

Processor $p$ delays a desired $\operatorname{Del}\left(Q^{p}, x\right)$ event until each processor that holds an $x$-token sent by $p$ has either returned the $x$-token to $p$ or has been accurately suspected by $p$ as faulty. Thus an $x$-token from $p$ not only informs another processor that $x$ has been added to $Q^{p}$, but also acts as a guarantee that $x$ is still a member of $Q^{p}$. A processor $q$ that chooses to hold an $x$-token from $p$ is assured that $p$ will delay the removal of $x$ from $Q^{p}$ until after $q$ has returned the $x$-token sent by $p$. By holding an $x$-token from $p, q$ delays the occurrence of a $\operatorname{Del}\left(Q^{p}, x\right)$ event at $p$, until $q$ determines that the $\operatorname{Del}\left(Q^{p}, x\right)$ event does not violate OQA-Safety. The following rules identify when it is safe

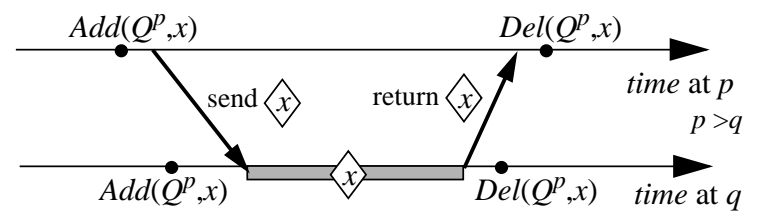

Figure 2: $q$ receives, holds, and returns matched $x$-token from $p$.

for $q$ to return an $x$-token to $p$.

An $x$-token held by $q$ is a matched token if and only if $x$ is currently in $q$ 's quorum $Q^{q}$. When $q$ holds any matched token received from $p$, then $q$ guarantees that $Q^{p}$ intersects $Q^{q}$. Figure 2 shows that processor $q$ holds a matched $x$-token from $p$ during the period when the timeline of $q$ is shaded. $Q^{p} \cap Q^{q}$ is guaranteed to contain processor $x$ while $q$ holds the matched $x$-token. The arrows indicate the exchange of the $x$-token via interprocessor messages. To ensure that $Q^{p} \cap Q^{q} \neq \varnothing$ remains satisfied, processor $q$ seeks to hold, at all times, at least one matched token from each other processor $p$. Because each two processors $\{p, q\}$ cooperate as peers to guarantee $Q^{p} \cap Q^{q} \neq \varnothing$, each pair of quorums are maintained with a non-empty intersection.

Processor $q$ must not take an action that results in the loss of all matched tokens that it holds from any $p$; otherwise, $Q^{p} \cap Q^{q} \neq \varnothing$ could be violated. If $p$ requests that $q$ return $p$ 's $x$-token, then $q$ can return the $x$-token immediately if it is not a matched token. Otherwise, the return of a matched $x$-token to $p$ must be delayed until $q$ holds some matched $y$-token from $p$, such that $y \neq x$. Processor $q$ must also delay local $\operatorname{Del}\left(Q^{q},-\right)$ events based on the status of its held quorum tokens. If $q$ holds a matched $x$-token from $p$ and wants to remove $x$ from $Q^{q}, q$ must delay the desired $\operatorname{Del}\left(Q^{q}, x\right)$ event until it holds some matched $y$-token from $p$, such that $y \neq x$.

Consider the example token exchange given in Figure 3 . Prior to the times indicated by the dashed line, $x$ is contained in $Q^{p}$ and $Q^{q}$, and $q$ has received an $x$-token sent by $p$. Both processors decide to replace $x$ with $y$ in their quorums, at the times marked by the dashed line, due to changes in $Q A$-desired ${ }^{p}$ and $Q A$-desired ${ }^{q}$ resulting from the failure of $x$ or the recovery of $y$. To satisfy OQA-Progress, $\operatorname{Del}(-, x)$ and $A d d(-, y)$ events are required to occur at both $p$ and at $q$. QADAPT executes the desired $\operatorname{Add}(-, y)$ events without delay. After the $\operatorname{Add}\left(Q^{p}, y\right)$ event, processor $p$ sends a message to $q$ that (1) gives a $y$-token to $q$ and (2) requests that $q$ return the $x$-token that $p$ sent earlier. The desired $\operatorname{Del}\left(Q^{p}, x\right)$ event is delayed by $p$ until $q$ has returned the $x$-token. Processor $q$ cannot immediately comply with

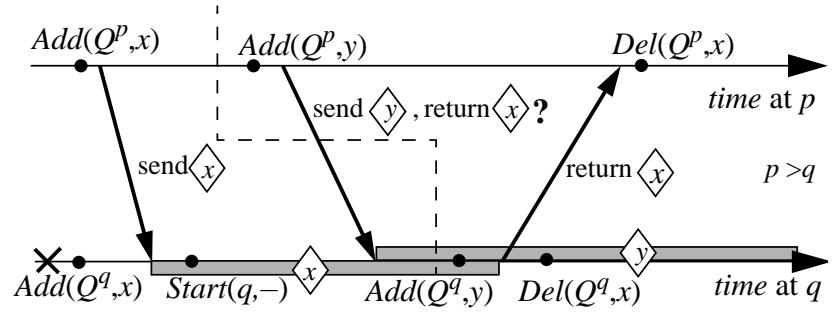

Figure 3: $x$ safely replaced with $y$ in $Q^{p}$ and $Q^{q}$. 
$p$ 's request that $q$ return the $x$-token, since the $x$-token is the only matched token that $q$ holds from $p$. At the time when $q$ receives $p$ 's request, $y$ has not been added to $Q^{q}$, so the $y$-token given by $p$ is not a matched token for $q$. Subsequent to receiving the $y$-token from $p, q$ decides that it should execute $\operatorname{Del}\left(Q^{q}, x\right)$ and $\operatorname{Add}\left(Q^{q}, y\right)$ events. The $\operatorname{Del}\left(Q^{q}, x\right)$ event cannot occur prior to the $\operatorname{Add}\left(Q^{q}, y\right)$ event; otherwise, the $\operatorname{Del}\left(Q^{q}, x\right)$ event occurs when $x$ is the only processor that $q$ can identify in $Q^{p} \cap Q^{q}$. After $q$ executes the $\operatorname{Add}\left(Q^{p}, y\right)$ event, the $y$-token held by $q$ becomes a matched token, allowing $q$ to identify $y \in Q^{p} \cap Q^{q}$. This enables $q$ to comply with $p$ 's earlier request and return the $x$-token to $p$. After the $\operatorname{Add}\left(Q^{p}, y\right)$ event $q$ can also proceed with the desired $\operatorname{Del}\left(Q^{q}, x\right)$ event.

For every pair of non-faulty processors in $\mathrm{S}_{\text {mutex }}$, QADAPT ensures that one processor in the pair assumes the role of always holding a matched token from the other processor. QADAPT assigns the role of token-holder to the processor with the lower identifier in each processor pair. Thus each processor is required to send quorum tokens to any other processor with an identifier less than or equal to than its own, and each processor must seek to always hold at least one matched token from any processor with a higher identifier than its own.

\subsection{Quorum Token Data Structures}

A more formal description of QADAPT follows. The data structures defined below are used to manage the exchange of quorum tokens and to identify the matched quorum tokens held by a processor. At each processor $p$, a $t$ _held_from ${ }^{p}$ table exists to keep track of the quorum tokens held by $p$. Each table entry is a list of tokens held by $p$; the index of the entry identifies the processor that sent the tokens to $p$. For example, the second entry of the $t \_$held_from ${ }^{p}$ table in Figure 4(a) indicates that processor $q$ sent to $p$ an $r$-token and an $s$-token, and that $p$ has not yet returned either of those tokens to $q$. The first entry of the same table indicates that $p$ has sent its own $p$ - and $q$-tokens to itself.

The $t \_s e n t \_t o^{p}$ table at $p$ indicates the processors to which $p$ has sent quorum tokens that have not yet been returned to $p$. The second entry in Figure 4(b) indicates that $p$ sent an $r$-token to $q$, and $q$ has not yet returned the token. The $t \_s e n t \_t o^{p}[q]$ entry indicates to $p$ that $q$ might be currently holding the $r$-token; in fact, $q$ might not yet have received the message conveying the $r$-token, or $q$ might have already attempted to return the $r$-token to $p$ by sending a message that $p$ has not yet received. It is also possible that $q$ has failed, losing the state that recorded its receipt of the $r$-token, and that $p$ has not yet suspected $q$ 's failure. (a)

\begin{tabular}{|c|}
\hline t_held_from \\
\hline$t$ held_from $[p]=\{p, q\}$ \\
$t$ _held_from $[q]=\{r, s\}$ \\
$t$ _held_from $[r]=\{q\}$ \\
$:$
\end{tabular}

(b)

\begin{tabular}{|c|}
\hline t_sent_to \\
\hline$t$ sent_to $[p]=\{p, q\}$ \\
$t$ _sent_to $[q]=\{r\}$ \\
$t$ _sent_to $[r]=\{t\}$ \\
$:$
\end{tabular}

Figure 4: Example data structures.

\subsection{Token Exchange Messages and Procedures}

Tokens are exchanged via TOKENS messages. Each TOKENS message $M$ has parameters grants and returns, denoted grants $\langle M\rangle$ and returns $\langle M\rangle$, that each contain a list of processor identifiers. A TOKENS $($ grants $\langle M\rangle$, returns $\langle M\rangle, \ldots$ ) message $M$ sent by $p$ to $q$ has a $\operatorname{grants}\langle M\rangle$ parameter equal to the list of quorum tokens that $p$ currently wants $q$ to hold. The returns $\langle M\rangle$ parameter identifies which, if any, tokens $p$ wishes to return to $q$. Any processors identified in the grants parameter must be contained in $Q^{p}$, and any tokens indicated in returns must have been previously received from $q$ by $p$. Processor $p$ implicitly requests that $q$ return a $y$-token by sending a TOKENS message $M$ to $q$ such that $y \notin$ grants $\langle M\rangle$. Since QADAPT requires any $p$ to either send tokens to $q \neq p$, or to receive tokens from $q$, but not both, $p$ leaves either the grants field or the returns field empty when it sends a TOKENS message to a different processor.

The following data structures are used to track the status of quorum token exchanges. The t_granted_to and t_granted_by tables use the same format as the $t_{-}$sent_to and $t \_h e l d \_$from tables. The value of the $t \_$granted_to ${ }^{p}[q]$ entry is always the same as the grants parameter of the TOKENS message most recently sent by $p$ to $q$. If $x \in t \_s e n t \_t o^{p}[q]$ but $x \notin t \_g r a n t e d \_t o^{p}[q]$, this indicates that $p$ has requested that $q$ return an $x$-token and is still waiting to learn that $q$ has returned the $x$-token. Conversely, t_granted_by ${ }^{q}[p]$ identifies quorum tokens received by $q$ from $p$, which $p$ has not yet asked $q$ to return. If $x \in t \_h e l d \_f r o m{ }^{q}[p]$ but $x \notin t \_g r a n t e d \_b y^{q}[p]$, this indicates $q$ has learned that $p$ requested the return of an $x$-token, but $q$ has not yet decided to return the $x$-token. The variable current_ $Q A^{p}$ stores the most recent desired quorum assignment provided to $p$ via the value of $Q A$-desired ${ }^{p}$, and old_QA ${ }^{p}$ stores the previous value of $Q A$-desired ${ }^{p}$.

The routines given in Figure 5 specify the QADAPT algorithm in pseudocode that is executed at each processor $p \in \mathrm{S}$. Two types of procedures are given. The notation [Action] is used for a procedure that is invoked when some local action occurs, where Action represents the delivery of a particular message to processor $p$, a change in the status of $p$ 's failure suspector, or a change in the status of $Q A$-desired" . A procedure denoted "PROC Procname" is invoked as a subroutine by a "DOPROC Procname" statement. The "send $(M, q)$ " action causes message $M$ to be sent by $p$ to processor $q$. The superscript " $p$ " is optionally omitted from local data structures, such that $t$ _held_from is a reference to $t \_h e l d$ from $^{p}$. Each procedure is assumed to execute atomically, without interruption by concurrent processing.

Procedure Update_Tokens control the exchange of TOKENS messages. The t_granted_to ${ }^{p}$ and $t \_s e n t \_t o^{p}$ tables are updated in lines 7.8-7.11 and 7.14-7.16, based on the current desired quorum assignment. Tokens that must be returned to other processors are determined in lines 7.4-7.7. Line 7.5 is the test that delays the return of a token if returning the token could lead to an OQA-Safety violation. A TOKENS message is sent (lines $7.14,7.18$ ) to any $j$ 
to convey updates that are made to $t \_g r a n t e d \_t o^{p}[j]$ or $t \_h e l d \_$from $^{p}[j]$. A single TOKENS message may indicate that new tokens have been sent to $j$ and may also implicitly request that $j$ return tokens previously sent by $p$. Upon receipt of a TOKENS message from processor $j, p$ updates $t \_$granted_by $^{p}[j], t \_h e l d \_$from $^{p}[j]$ and $t \_s e n t \_t o^{p}[j]$ (lines $2.1-2.3)$. If $j$ has requested the return of tokens, then the procedure call made to Update_Tokens (see line 6.8, which is invoked via the procedure call of 2.7) will cause the tokens to be returned immediately, if doing so will not violate OQA-Safety.

Procedure Update_Quorum is invoked each time $Q A$-desired ${ }^{p}$ is updated or a TOKENS message is received. Update_Quorum adds desired processors to the local quorum $Q^{p}$ without delay (lines 6.2-6.3) and removes a processor $k$ from $Q^{p}$ (lines 6.5-6.7) only if (1) there is no processor $x$ such that a matched $k$-token is $p$ 's only guarantee that $Q^{p} \cap Q^{x}$ is not empty, and if (2) $p$ knows of no unreturned $k$-tokens that it previously sent to other processors.

Since $p$ may repeatedly add and remove $x$ to or from $Q^{p}$, logical timestamps [21] are used to distinguish between multiple $x$-tokens sent by $p$ to processor $j$, and to detect when TOKENS messages that convey an $x$-token and request the $x$-token's return are received out of order. Each processor maintains a local variable $m y \_$time $^{p}$ that is incremented each time $p$ sends a message. The value of $m y \_t i m e^{p}$ is included with each message sent by $p$. Each quorum token that is received by $p$ is held (added to t_held_from ${ }^{p}$ ) along with the timestamp of the TOKENS message that delivered the token to $p$. For simplification, references to message and token timestamps and to $m y \_$time $e^{p}$ are omitted from the procedures given in Figure 5. Complete details of timestamps are given in [5].

\subsection{Tolerating Processor Failures (Stop Events)}

It is assumed that QADAPT executes using volatile local storage at each $p$. OQA-Progress is threatened if $p$ fails or leaves $S$ while holding quorum tokens from other processors. Lost tokens can prevent processors from ever deleting members of their quorums. Since failure suspectors are not guaranteed to be accurate when communication is not occurring, it is possible for $p$ to fail and subsequently recover without other processors suspecting that $p$ failed. To prevent $p$ from never returning some quorum token that must be returned to enable OQA-Progress, the has_sent parameter of the TOKENS message in lines 7.17-7.18 informs $p$ of tokens that $p$ held when it previously failed. The response given in lines 2.5-2.6 ensures that $p$ eventually returns tokens, if necessary for OQA-Progress, after $p$ recovers from a failure. If $p$ fails while holding tokens and never recovers, then any processor $q$ that remains non-faulty must accurately suspect that $p$ failed. Lines 4.0-4.4 in Figure 5 guarantee that $q$ eventually attempts to communicate with $p$, if it is necessary for $q$ to accurately suspect $p$ 's failure. Lines 3.0-3.2 ensure that OQA-Progress is not blocked by tokens that are never returned by faulty $p$. Upon accurately identifying $p$ as

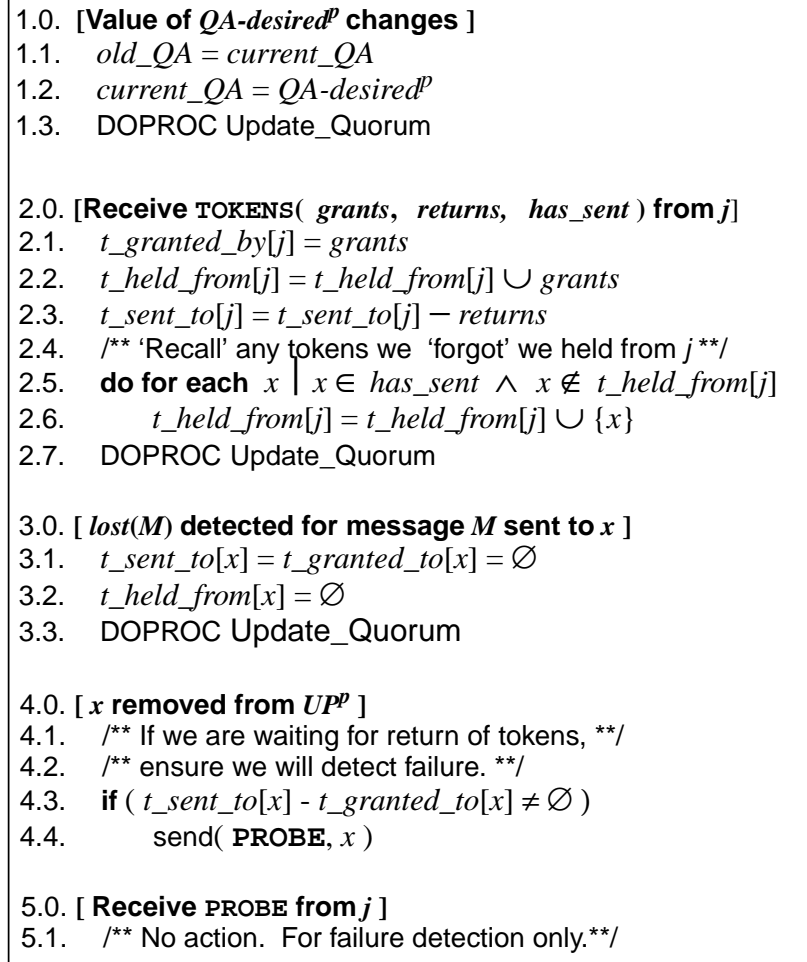

6.0. PROC Update_Quorum

6.1. ${ }^{* *}$ Perform desired $A d d\left(Q^{p},-\right)$ 's (always safe) ${ }^{* *} /$

6.2. do for each $x \mid x \in$ current_QA: $Q^{p} \wedge x \notin Q^{p}$

6.3. $Q^{p}=Q^{p}+\{x\}$

6.4. ${ }^{* *}$ Perform desired $\operatorname{Del}\left(Q^{p},-\right)$ 's (only if safe) ${ }^{* *} /$

6.5. do for each $k \mid k \in Q^{p^{2}} \wedge k \notin$ current_QA: $Q^{p}$

6.6. if $\left(\left(\nexists x \mid t_{-}\right.\right.$held_from $\left.[x] \cap Q^{p}=\{k\}\right) \wedge$

6.7. $Q^{p}=Q^{p}-\{k\}$ $\left\{x\left\lceil k \in t \_s e n t \_t o[x]\right\}=\varnothing\right)$

6.8. DOPROC Update_Tokens

6.9. DOPROC Check_Join_Status

\subsection{PROC Update_Tokens}

7.1. ${ }^{* *}$ Determine set of tokens to grant or return. ${ }^{* * /}$

7.2. ${ }^{* *}$ Generate a TOKENS message if needed. ${ }^{* *} /$

7.3. do for each $j$ old_QA: $Q^{J} \cup$ current_QA: $Q^{j} \neq \varnothing$

7.4. do for each $x\left\lceil x \in t\right.$ theld_from $[j] \wedge x \notin t \_g r a n t e d \_b y[j]$

7.5. if $\left(\left(Q^{p} \cap t \_h e l d \_\right.\right.$from $\left.\left.[j]\right) \supset\{x\}\right)$

7.6. $\quad$ returns $=$ returns $\cup\{x\}$

7.7.

7.8.

7.9.

7.10.

7.11 .

7.12 .

7.13.

7.14 .

7.15.

7.16 .

7.17 .

7.18.

$$
\begin{aligned}
& \text { if }(p \geq j) \\
& \text { grants }=\text { current_QA: } Q^{p} \cap \text { current_QA: } Q^{j} \\
& \text { else if }(j>p) \\
& \text { grants }=\varnothing \\
& { }^{* *} \text { If status of granted/held tokens changed, }{ }^{* *} / \\
& { }^{* *} \text { send TOKENS message to } j{ }^{* *} / \\
& \text { if }\left(\text { grants } \neq t \_ \text {granted_to }[j] \vee \text { returns } \neq \varnothing\right) \\
& t \_s e n t \_t o[j]=t \_s e n t \_t o[j] \cup \text { grants } \\
& t \_ \text {granted_to }[j]=\text { grants } \\
& \text { has_sent }=t \_s e n t \_t o[j] \\
& \text { send( TOKENS( grants, returns, has_sent, }), j \text { ) }
\end{aligned}
$$$$
t \_h e l d \_ \text {from }[j]=t \_h e l d \_ \text {from }[j]-\{x\}
$$

Figure 5: QADAPT algorithm: Token and quorum updating routines exectued at $p$. 
faulty, $q$ acts as if $p$ returns all tokens that $q$ sent to $p$. Lines 3.1-3.2 are executed by $q$ only when $q$ knows that its suspicion that $p$ failed is accurate, i.e., when $q$ attempts communication with $p$. If a non-faulty processor $p$ chooses to voluntarily leave the set $\mathrm{S}_{\text {mutex }}$, by setting mutex $^{p}$ to FALSE, then $p$ must send TOKENS messages that return all of its held quorum tokens.

QADAPT must prevent OQA-Safety from being violated when $p$ sends a quorum token to processor $q$, and then $p$ fails or leaves $\mathrm{S}$. If $p$ subsequently recovers or rejoins $\mathrm{S}$, then any token $p$ sent before failing becomes a ghost token. An $x$-token held by $q$ from $p$ is a ghost token when $x \notin t \_s e n t \_t o^{p}[q]$; an $x$-token held by $q$ from $p$ is live when $x \in t \_s e n t \_t o p[q]$. A matched ghost token held by $q$, unlike a matched live token, provides no guarantee to $q$ that $Q^{p} \cap Q^{q} \neq \varnothing$ is true. Since $p$ cannot identify a ghost token held by $q, p$ may execute a $\operatorname{Del}\left(Q^{p}, x\right)$ event if a ghost $x$-token is the only matched token $q$ holds from $p$. The timestamps included with each message and stored with each quorum token are used to detect ghost tokens. Upon receiving a TOKENS message from $p, q$ detects and eliminates ghost tokens, since the timestamp stored with a ghost-token is older than the timestamp received from $p$ with the TOKENS message. For simplification, the steps for detecting and eliminating ghost tokens are omitted from the pseudocode. Further details are given in [5].

\subsection{Processor Joins and Recoveries (Start events)}

Processor $p$ may fail or leave $\mathrm{S}$ and subsequently desire to rejoin $S_{\text {mutex }}$, after recovering or rejoining $S$. Before $p$ succeeds at joining $\mathrm{S}_{\text {mutex }}$ via a $\operatorname{Start}(p,-)$ event, $p$ is said to be executing the join phase of QADAPT, or joining. A brief synopsis of join phase operation is given here; see [5] for full details. When $p$ starts the join phase, it is assumed that $p$ has no record of any previously sent or held quorum tokens. The join phase establishes that for each $q \in \mathrm{S}_{\text {mutex }}$, either $p$ holds a matched token from $q$, or $q$ holds a matched token from $p$. The final step of the join phase at processor $p$ occurs when $p$ sets the value of mutex $^{p}$ to TRUE. After $p$ completes the join phase it can safely issue request messages to processors in $Q^{p}$. The timelines in Figure 3 also serve to illustrate the required sequence of events prior to a Start event at $q$, for each $p$ that is already in $\mathrm{S}_{\text {mutex }}$ when $p$ joins $\mathrm{S}_{\text {safe }}$. The "X" on $q$ 's timeline indicates the time at which $q$ begins executing QADAPT, after recovering from a failure or joining $\mathrm{S}$. Processor $q$ 's join phase terminates with the $\operatorname{Start}(q,-)$ event.

Processors have no mechanism that accurately identifies the current members of $\mathrm{S}_{\text {mutex }}$, since the current values of $Q A$-desired ${ }^{p}$ and $U P^{p}$ give $p$ only a hint at the current membership of $\mathrm{S}$ and $U P(\mathrm{~S})$. Joining processor $p$ must establish that a quorum token has been exchanged between $p$ and $q$, for every $q \in \mathrm{S}_{\text {mutex }}$, prior to the $\operatorname{Start}(p,-)$ event that terminates $p$ 's join phase. Although failure suspectors do not accurately identify the current membership of $\mathrm{S}_{\text {mutex }}$, each processor $q \in \mathrm{S}_{\text {mutex }}$ can identify a set of processors TC_suspects ${ }^{q}$ that must currently be a superset of $\mathrm{S}_{\text {mutex }}$, since $q$ must have either received or sent a quorum token to or from each other member of $\mathrm{S}_{\text {mutex }}$. TC_suspects ${ }^{q}$ is the set of processors such that $q$ holds a matched token from, or has sent a token to, every member of TC_suspects ${ }^{q}$. If some processor $z$ is in $\mathrm{S}_{\text {mutex }}$ and $q$ is also in $\mathrm{S}_{\text {mutex }}$, then $z$ must be in $T C \_$suspects ${ }^{q}$. Joining processor $p$ creates its own set $T C \_$suspects $^{p}$ by adding processors to $T C_{-}$suspects $^{p}$ that are contained in the TC_suspects ${ }^{q}$ set of each processor $q \in \mathrm{S}_{\text {mutex }}$ that $p$ is able to identify. Eventually, $p$ is able to determine that $T C_{-}$suspects ${ }^{p} \supseteq \mathrm{S}_{\text {mutex }}$ is true, when $p$ has learned the value of TC_suspects ${ }^{q}$ for every $q \in T C \_$suspects ${ }^{p}$. Processor $p$ delays a desired $\operatorname{Start}(p,-)$ event until it learns that each $q \in T C_{-}$suspects $^{p} \supseteq \mathrm{S}_{\text {mutex }}$ has either received a matched token from $p$ or has sent a matched token to $p$. TOKENS messages exchanged during the join phase ensure that all processors are informed of tokens they held prior to any failure, and that all ghost tokens from $p$ are eliminated by processors in $\mathrm{S}_{\text {mutex }}$, prior to $p$ 's completion of the join phase.

\subsection{QADAPT Correctness}

The proof is given in [5] that QADAPT guarantees OQA-Safety and OQA-Progress, given the system assumptions of Section 2. At least one processor must remain non-faulty and a member of $S_{\text {mutex }}$ during any period when two or more processors concurrently execute the join phase. The following definition is central to the correctness argument for the QADAPT algorithm.

Definition (token-consistency): Two processors $j$ and $k$, $j>k$, are token-consistent when the following proposition is satisfied

$$
Q^{j} \cap Q^{k} \cap t \text { _sent_to }{ }^{j}[k] \cap t \_h e l d \_ \text {rrom }^{k}[j] \neq \varnothing \text { (TC) }
$$

or when either $j$ or $k$ is faulty. Processor $p$ is token-self-consistent only if (TC) is satisfied when $j=k=p$, or if $p$ is faulty. A set $T \subseteq \mathrm{S},|T|>0$, is a token-consistent set if and only if each processor in $T$ is self-token-consistent, and each pair of processors in $T$ are token-consistent. $T C_{i}(X)$ is the proposition that set $X$ is token-consistent when the configuration is $C_{i}$.

The following theorem, proved in [5], shows that QADAPT guarantees OQA-Safety by maintaining token-consistency for all processors in $\mathrm{S}_{\text {mutex }}$. $T C_{i}\left(\mathrm{~S}_{\text {mutex }}\left(C_{i}\right)\right)$ is shown to be true for all $i>0$, given that $\mathrm{S}_{\text {mutex }}\left(C_{i}\right)$ are the processors in $\mathrm{S}_{\text {mutex }}$ when the configuration is $C_{i}$.

Theorem 1. Given that $T C_{0}\left(\mathrm{~S}_{\text {mutex }}\left(C_{0}\right)\right)$ is satisfied, then $T C_{x}\left(\mathrm{~S}_{\text {mutex }}\left(C_{x}\right)\right)$ is true for all $x>0$.

From Theorem 1, the definition of OQA-Safety, and expression (TC), it follows that QADAPT preserves OQA-Safety. The rules governing token exchange ensure that, once satisfied, $T C(\{j, k\})$ remains satisfied while neither $j$ nor $k$ executes a Stop event. Stop events cannot jeopardize OQA-Safety. Once a processor $p$ has left $\mathrm{S}_{\text {mutex }}$ and wishes to rejoin, it is required to complete the join phase, which ensures that $T C_{i}\left(p \cup \mathrm{S}_{\text {mutex }}\left(C_{i}\right)\right)$ is satisfied when $p$ executes a $\operatorname{Start}_{i}(p,-)$ event. Eventual termination of the join phase is also proved in [5]. 


\section{QADAPT Execution Costs}

The execution cost of an OQA algorithm is determined by a large number of execution parameters, including distribution of quorum sizes, quorum topology, and the pattern of processor failures. To analyze the cost of QADAPT, a general round of quorum adaptation called an $\alpha$-step is considered. An $\alpha$-step is a transition from one valid quorum assignment to a different valid quorum assignment, such that exactly one processor $r$ is removed from each of the $K$ quorums that $r$ appears in. Before its removal from the quorum assignment, $r$ is the only processor in the intersection of any two quorums containing $r$. Thus for every pair of quorums that contain $r, r$ is replaced by some other processor. As shown in [4], an $\alpha$-step requires exactly $K \operatorname{Del}(-, r)$ and $K A d d$ events. One $D e l$ and one $A d d$ must occur at each processor whose quorum contains $r$ prior to the $\alpha$-step.

QADAPT requires $K(K-1)$ TOKENS messages to complete an $\alpha$-step, corresponding to a pair of messages exchanged between every distinct pair of processors that must both execute $\operatorname{Del}(-, r)$ events. Figure 3 illustrates the exchange of two messages to replace a processor $(x)$ with another $(y)$ in the intersection of two quorums. By the lower bound analysis given in [4], the number of messages required by QADAPT for an $\alpha$-step is minimal. If the quorum assignment is a balanced assignment [25], such that $K$ is $\mathrm{O}(\sqrt{|\mathrm{S}|})$, then QADAPT requires $\mathrm{O}(|\mathrm{S}|)$ messages to eliminate one faulty processor from each quorum.

The best case delay for completing an $\alpha$-step is $2 d$, where $d$ is the delay to send and deliver a message. In the worst case, the number of messages required by the join phase before a processor can execute a Start event is $3 \mathrm{~N}$, and the delay to complete the join phase is $4 d$. The full analysis is given in [5].

Measurements are given below for an experimental implementation of QADAPT at Carnegie Mellon University. The delay of executing a set of concurrent $\alpha$-steps, with $K=\sqrt{|S|}$ as in [25], was measured while QADAPT executed as background processes on a testbed containing 50 networked Unix workstations. Reliable messages were approximated by using unreliable messages with an acknowledge-or-time-out/retry protocol. The background processing load during experimentation was high, since

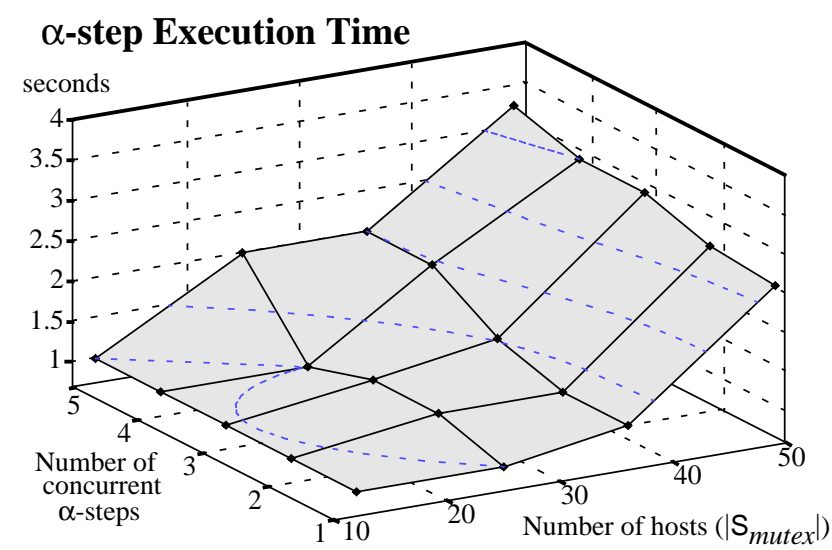

Figure 6: QADAPT execution delay on networked workstations. most of the workstations are located in a public laboratory and were continuously shared by several users. ${ }^{1}$ Execution times for completion of a set of induced $\alpha$-steps were approximately 1 second for 25 or fewer hosts connected on a single switched ethernet segment, and approximately 3 seconds for 50 hosts on two switched ethernet segments with an intermediate campus router. The graph in Figure 6 shows how execution time increased as the number of concurrent $\alpha$-steps and the value of $\left|S_{\text {mutex }}\right|$ were increased.

\section{Summary and Discussion}

This paper presents an efficient, decentralized, and fault-tolerant solution to the on-line quorum adaptation (OQA) problem. OQA is the problem of modifying quorums so that the intersection property required for mutual exclusion safety is continuously preserved. An OQA algorithm provides the ordering of events that is needed to safely install any desired valid quorum assignment. An OQA solution is applicable to any dynamic optimization of quorums, whether carried out for fault tolerance, for system reconfiguration, or for performance optimization.

A distributed OQA algorithm called QADAPT is given that tolerates up to $N$-1 simultaneous failures among $N$ processors and requires no view-synchronous communication mechanism to ensure that modifications to quorums are correctly ordered. Although a view-synchronous communication model [7] greatly simplifies the solution of a distributed coordination problem such as OQA, view-synchrony results in potentially unnecessary synchronization costs that are avoided by the unordered system model that QADAPT assumes. The message passing cost of QADAPT is shown to be optimal, based on the lower bound analysis in [4], requiring approximately $N$ point-to-point messages to remove one failed processor from $\sqrt{N}$ quorums.

QADAPT improves upon previous dynamic quorum adaptation approaches by making no restrictions on the number of simultaneously faulty processors or the topology of the quorum assignment. QADAPT allows the set of desired quorums to be computed based on temporarily incorrect or inconsistent failure suspectors. When failure suspectors are incorrect or inconsistent, a processor's access to the critical resource may be delayed, or unnecessary quorum adaptation steps may be executed, but mutual exclusion safety is never violated. Work in progress includes experimental analysis of the message passing costs of QADAPT, under a variety of quorum topologies and adaptation patterns.

The presentation assumes that a communication network partition cannot prevent two non-faulty processors from communicating. The assumption can be relaxed somewhat, if a single "primary partition" [3] is assumed to exist at all times, such that only processors in the primary partition attempt to execute the mutual exclusion or on-line quorum adaptation protocols. Progress and safety can be guaranteed for the subset of processors in the primary partition, if all processors not in the primary partition are sus-

\footnotetext{
1 The implementation focused on demonstrating the scalability of QADAPT, rather than on minimizing execution time.
} 
pected by those in the primary partition (perhaps incorrectly) to be faulty. In the case of partitions, ensuring FS-Comm-Accuracy is not possible [30]. Establishing a primary partition in a real system is problematic, but is necessary for any distributed mutual exclusion protocol [12] that tolerates partitions.

The quorum intersection property is used in the context of mutual exclusion to limit concurrency. In database systems, quorum assignments have been used to increase concurrency when reading and writing replicated data $[14,15,16,24,33]$. In such cases the quorum intersection property provides mutual consistency of read and write operations rather than exclusive access to a resource. This work focuses on the mutual exclusion problem, but the results are also applicable to other quorum-based distributed coordination problems, as shown in [5].

\section{References}

[1] Amir, Y. and Wool, A., "Evaluating Quorum Systems over the Internet," Proc. of 26th Intl. Symp. on Fault-Tolerant Computing, Sendai, Japan, pp. 26-35.

[2] Agrawal, D. and El Abbadi, A., "An Efficient and Fault-Tolerant Solution for Mutual Exclusion," ACM Trans. on Computer Systems, 9(1), Feb. 1991, pp. 1-20.

[3] Bartoli, A. and Babaolgu, Ozalp, "Selecting a Primary Partition in Partitionable Asynchronous Distributed Systems," Proc. 16th Symp. on Reliable Distributed Systems, Durham, North Carolina, Oct. 1997, pp. 138-145.

[4] Bearden, M. and Bianchini, R., "The Synchronization Cost of On-line Quorum Adaptation," Proc. 10th Intl. Conf. on Parallel and Distributed Computing Sys., New Orleans, Louisiana, Oct. 1997, pp. 598-605.

[5] Bearden, M., "Fault-tolerant On-line Adaptation of Quorum Assignments for Decentralized Coordination" Ph.D. Thesis, Dept. of Electrical and Computer Engineering, Carnegie Mellon University, Pittsburgh, Pennsylvania, Jan. 1998.

[6] Bianchini, R. and Buskens, R., "Implementation of On-Line Distributed System-Level Diagnosis Theory," IEEE Transactions on Computers, 41(5), May 1992, pp. 616-626.

[7] Birman, K., "The Process Group Approach to Reliable Distributed Computing," Communications of the ACM, 36(12), Dec. 1993, pp. 37-53.

[8] Bouabdallah, A., "On Mutual Exclusion in Faulty Distributed Systems," Operating Systems Review (ACM SIGOS), 28(1), Jan. 1994, pp. 80-87.

[9] Chen, H. and Tang, H., "An Efficient Method for Mutual Exclusion in Truly Distributed Systems," Proc. 14th Intl. Conference of Distributed Computing Systems, Poznan, Poland, 1994, pp. 97-104.

[10] Cheung, S. Ammar, M., and Ahamad, M., "The Grid Protocol: A High Performance Scheme for Maintaining Replicated Data," IEEE Trans. on Knowledge and Data Engineering, 4(6), Dec. 1992, pp. 582-92.

[11] Cheriton, D. and Skeen, D., "Understanding the Limitations of Causally and Totally Ordered Communication," 14th ACM Symposium on Operating Systems Principles, Dec. 1993, Asheville, North Carolina.

[12] Coan, B., Oki, B., and Kolodner, E., "Limitations on Database Availability When Networks Partition, " Proc. 5th Symp. on Principles of Distributed Computing, Calgary, 1986, pp. 187-194.

[13] Dijkstra, E. W., "Solution of a Problem in Concurrent Programming Control," Communications of the ACM 8(9), 1965 , p. 569.
[14] Garcia-Molina, H. and Barbara, D., "How to Assign Votes in a Distributed System," Journal of the ACM, 32(4), Oct. 1985, pp. 841-860.

[15] Gifford, D., "Weighted Voting for Replicated Data," Proceedings of the Seventh ACM Symposium on Operating Systems Principles, Dec. 1979, pp. 150-159.

[16] Helal, A., Heddaya, A., and Bhargava, B., Replication Techniques in Distributed Systems, Kluwer Academic Publishers, Boston, 1996. $156 \mathrm{pp}$.

[17] Herlihy, M., "Dynamic Quorum Adjustment for Partitioned Data," ACM Transactions on Database Systems 12(2), June 1987, pp. 170-94.

[18] Jajodia, S. and Mutchler, D., "Dynamic Voting Algorithms for Maintaining the Consistency of a Replicated Database," ACM Transactions on Database Systems, 15(2), June 1990, pp. 230-280.

[19] Johnson, D. and Raab, L., "Effects of Replication on Data Availability," Intl. Journal of Computer Simulation, 1(4), 1991, pp. 373-392.

[20] Kumar, A., "Hierarchical Quorum Consensus: A New Algorithm for Managing Replicated Data," IEEE Transactions on Computers, 40(9), Sep. 1991, pp. 996-1004.

[21] Lamport, L., "Time, Clocks, and the Ordering of Events in a Distributed System," Communications of the ACM, 21(7), July, 1978, pp. 558-565.

[22] Liu, M., Agrawal, D., and El Abaddi, A., "On the Implementation of the Quorum Consensus Protocol," Proc. Parallel and Distributed Computing Systems, Orlando, Florida, 1995.

[23] Lotem, E., Keidar, I., and Dolev, D., "Dynamic Voting for Consistent Primary Components," Proc. 16th ACM Symp. on Principles of Distributed Computing, Santa Barbara, California, Aug. 1997.

[24] Lynch, N. and Shvartsman, A., "Robust Emulation of Shared Memory Using Dynamic Quorum-Acknowledged Broadcasts," in Proc. 27th Intl. Symp. on Fault-Tolerant Computing Systems, Seattle, Washington, June 1997.

[25] Maekawa, M., "A $\sqrt{N}$ Algorithm for Mutual Exclusion in Decentralized Systems," ACM Transactions on Computer Systems 3(2), May 1985, pp. 145-159.

[26] Peleg, D. and Wool, A., "Crumbling Walls: A Class of High Availability Quorum Systems," Proc. 14th ACM Symp. on Principles of Distributed Computing, Ottawa, Ontario, 1995, pp. 120-9.

[27] Peleg, D. and Wool, A., "The Availability of Quorum Systems," Information and Computation, 123(2), Dec. 1995, pp. 210-223.

[28] Peleg, D. and Wool, A., "How to be an Efficient Snoop, or the Probe Complexity of Quorum Systems," Proc. 15th ACM Symp. on Principiples of Distributed Computing," Philadelphia, Pennsylvania, May 1996, pp. 290-9.

[29] Rangarajan, S. and Tripathi, S., "A Robust Distributed Mutual Exclusion Algorithm," Distributed Algorithms, Proceedings 5th Intl. Workshop, WDAG '91, Delphi, 1991, Springer-Verlag, pp. 295-308.

[30] Ricciardi, A., Schiper, A., and Birman, K., "Understanding Partitions and the 'No Partition' Assumption," Proc. 4th Intl. Workshop on Future Trends of Distributed Computing Systems, Lisbon, Sep. 1993, pp. 354-360.

[31] Sanders, B., "The Information Structure of Distributed Mutual Exclusion Algorithms," ACM Transactions on Computer Systems 5(3), Aug. 1987, pp. 284-299.

[32] Schneider, F., "Byzantine Generals in Action: Implementing Fail-Stop Processors," ACM Trans. on Computer Systems, 2(2), May 1984, pp. 145-154.

[33] Thomas, R., "A Majority Consensus Approach to Concurrency Control for Multiple Copy Databases," ACM Transactions on Database Systems, 4(2), June 1979, pp. 180-209. 